\title{
Dead Tumor Cells Expressing Infectious Salmon Anemia Virus Fusogenic Protein Favor Antigen Cross-Priming In Vitro
}

\author{
Jonathan Morales ${ }^{1 \dagger}$, Carlos Barrera-Avalos ${ }^{1 \dagger}$, Carlos Castro', Stephanie Castillo', \\ Claudio Barrientos', Claudia Robles-Planells', Ximena López', Ernesto Torres', \\ Margarita Montoya', Marcelo Cortez-San Martín', Denise Riquelme', Alejandro Escobar ${ }^{2}$, \\ Ricardo Fernández ${ }^{3}$, Mónica Imarai ${ }^{1}$, Daniela Sauma ${ }^{4}$, Leonel E. Rojo ${ }^{5}$, \\ Elias Leiva-Salcedo ${ }^{5}$ and Claudio Acuña-Castillo ${ }^{1 *}$ \\ 'Centro de Biotecnología Acuícola, Departamento de Biología, Facultad de Química y Biología, Universidad de Santiago de \\ Chile, USACH, Santiago, Chile, '2Instituto de Investigación en Ciencias Odontológicas, Facultad de Odontología, Universidad \\ de Chile, Santiago, Chile, ${ }^{3}$ Departamento de Salud, Universidad de Los Lagos, Osorno, Chile, ${ }^{4}$ Departamento de Biología, \\ Facultad de Ciencias, Universidad de Chile, Santiago, Chile, ${ }^{5}$ Departamento de Biología, Facultad de \\ Química y Biología, Universidad de Santiago de Chile, USACH, Santiago, Chile
} Sherven Sharma, VA Greater Los Angeles Healthcare System (NHA), United States

Reviewed by: Carlos Alfaro, Universidad de Navarra, Spain Kawaljit Kaur, University of California, Los Angeles, United States

${ }^{*}$ Correspondence: Claudio Acuna-Castillo claudio.acuna@usach.cl

tThese authors have contributed equally to this work.

Specialty section: This article was submitted to Cancer Immunity and Immunotherapy, a section of the journal Frontiers in Immunology

Received: 02 June 2017 Accepted: 04 September 2017 Published: 09 October 2017

Citation:

Morales J, Barrera-Avalos C, Castro C, Castillo S, Barrientos C, Robles-Planells C, López X, Torres E, Montoya M, Cortez-San Martín M, Riquelme D, Escobar A, Fernández $R$, Imarai $M$, Sauma $D$, Rojo $L E$, Leiva-Salcedo E and AcuñaCastillo C (2017) Dead Tumor Cells Expressing Infectious Salmon Anemia

Virus Fusogenic Protein Favor Antigen Cross-Priming In Vitro.

Front. Immunol. 8:1170 doi: 10.3389/fimmu.2017.01170
Antigen cross-presentation is a crucial step in the assembly of an antitumor immune response leading to activation of naive CD8 T cells. This process has been extensively used in clinical trials, in which dendritic cells generated in vitro are loaded with tumor antigens and then autotransplanted to the patients. Recently, the use of autologous transplant of dendritic cells fused with dying tumor cells has demonstrated good results in clinical studies. In this work, we generated a similar process in vivo by treating mice with dead tumor cells [cell bodies (CBs)] expressing the fusogenic protein of the infectious salmon anemia virus (ISAV). ISAV fusion protein retains its fusogenic capability when is expressed on mammalian cells in vitro and the CBs expressing it facilitates DCs maturation, antigen transfer by antigenpresenting cells, and increase cross-presentation by DCs in vitro. Additionally, we observed in the melanoma model that CBs with or without ISAV fusion protein reduce tumor growth in prophylactic treatment; however, only ISAV expressing CBs showed an increase CD4 and CD8 cells in spleen. Overall, our results suggest that CBs could be used as a complement with other type of strategies to amplify antitumor immune response.

Keywords: infectious salmon anemia virus, cross-priming, antitumor immune response, b16 melanoma, cell fusion

\section{INTRODUCTION}

Dendritic cells are the most important antigen-presenting cells (APCs); they are present at trace levels in the tissues and stand for less than $0.5 \%$ of circulating leukocytes. These cells activate naïve CD8 $\mathrm{T}$ cells by delivering antigens through cross-priming and this particular characteristic has been used in cancer immunotherapy clinical trials (1). Immunotherapy using dendritic cells in vitro requires a large amount of cells, these are obtained by differentiating monocytes or CD34+ progenitors with granulocyte-macrophage colony-stimulating factor and IL-4 (2). These cells can be loaded with tumor antigens and multiple techniques have been used for this purpose, including tumor-extracted RNA transfection, pulsing with tumor lysates, apoptotic body induction, peptides, tumor-derived exosomes, and heterokaryon-induction from tumor-dendritic cell fusion (3).

The antigen source for dendritic cells loading is important in the antitumor response; in prophylactic treatments in vivo, complete cell structures like exosomes induce a potent antitumor 
response $(4,5)$. The cytotoxic effect over the tumors induced by chemotherapeutic agents (6) generates an immunogenic cell death (ICD) and is linked with an antitumor immune response (7). In this context, animals inoculated with dying tumor cells undergoing ICD (induced by oxaliplatins) elicit tumor-specific immune responses protecting the animals against subsequent tumor challenges (8).

Immunogenic cell death enhances exogenous antigen crosspresentation in the context of MHC class I (9); in this regard, Li et al. (10) showed that starved HEK 293T cells, expressing the chicken egg albumin (OVA), undergoes macro-autophagy, presenting an enhanced OVA cross-presentation to CD8+ $\mathrm{T}$ cells (11). Furthermore, the inhibition of autophagy reduced the epitope-specific CD8+ T cell antigen cross-presentation (11).

Virotherapy induces tumor cell death and present potential advantages over conventional treatments (12). The type of cell death induced by this therapy is important and the mechanism is not well understood. In this regard, some types of virotherapy can generate ICD (13), through the induction of in vivo fusion between tumor cells (14). On the other hand, Hoffmann et al. (15) demonstrated that only the use of viral fusogenic membrane glycoproteins (FMGs) are enough to induce tumor cells fusion leading to a potent and localized tumor size reduction. Furthermore, B16 melanoma expressing the fusogenic membrane protein $\mathrm{G}$ from the vesicular stomatitis virus (VSV-G) improve the efficacy of weak allogeneic vaccines (16). These data suggest that ICD induced by FMGs could be a mechanism to improve tumor regression by increasing cross-priming.

In the infectious salmon anemia virus (ISAV), a member of the influenza virus family (17), the infection is initiated by receptor binding and internalization in endosomes; the viral and endosomal membrane is fused by a mechanism mediated by the ISAV fusion protein. In this context, ISA fusion protein expressed in tumor cell bodies (CBs) (dead cells) might be a good candidate to mediate the fusion between the $\mathrm{CB}$ and the phagosome or cellular membranes of the APCs, thus delivering antigens to the cytoplasm enhancing cross-priming. Here, we report that the prophylactic antitumor treatment using CBs, independent of the expression of ISAV fusion protein suggesting that CBs can be used as a complement with other antitumor strategies.

\section{MATERIALS AND METHODS}

\section{Animals and Cell Cultures}

Eight- to ten-week-old C57BL/6J (H2b) were obtained from the Universidad de Santiago de Chile animal facility. The animals were fed ad libitum with a 12/12 h light/dark cycle. All procedures were conducted in accord to guidelines on the recognition of pain, distress, and discomfort in experimental animals described by Morton and Griffiths, except for temperature evaluation (18). Protocols were reviewed and approved by the Ethics Committee of the Universidad de Santiago de Chile.

HEK293 (kindly provided by Dr. Andres Stutzin), MDCK (kindly provided by Dr. Monica Imarai), Raw264.7 (kindly provided by Dr. Maria Rosa Bono), and murine melanoma B16 (kindly provided by Dr. Flavio Salazar) cell lines were cultured in Dulbecco's modified Eagle's medium. Media was supplemented with $10 \%$ fetal bovine serum, $100 \mathrm{U} / \mathrm{mL}$ penicillin, and $100 \mu \mathrm{g} / \mathrm{mL}$ streptomycin and cells were kept at $37^{\circ} \mathrm{C}$ in a humidified atmosphere under $5 \% \mathrm{CO}_{2}$. Mouse bone marrow-derived dendritic cells (BM-dendritic cells) were generated as previously described (19).

\section{ISAV Fusion Protein and Transfections}

Fusion protein sequence was isolated from an ISAV outbreak in Chile, the fusion protein is encoded in the segment 5 of the ISAV genome. The ISAV fusion protein gene sequence was synthesized by Genscript (NJ, USA) and subcloned from pUC57 using primers containing the sequence for EcoRI and XhoI for pIRES, and HindIII and XhoI for pCDNA3.1.

HEK293, MDCK, and B16 cell lines were transfected with pIRES-ISAV or pcDNA3.1-ISAV using Lipofectamine 2000 (Thermofisher, USA) according to the manufacturer's recommendations. Stably transfected cells were selected and maintained with $0.4 \mathrm{mg} / \mathrm{mL}$ G418.

\section{CBs Generation}

Infectious salmon anemia virus-transfected or wild-type B16 or HEK293 cells were grown until 70\% confluence, and then they were washed with PBS and deprived of nutrients by switching culture media to PBS containing $2.5 \mu \mathrm{g} / \mathrm{mL}$ fungizone and $10 \mu \mathrm{g} /$ $\mathrm{mL}$ gentamycin for 1 week at $37^{\circ} \mathrm{C}$ in a humidified atmosphere under $5 \% \mathrm{CO}_{2}$. At day 7 , the supernatant was centrifuged at $300 \mathrm{~g}$ and the pellet was stored in PBS at $4^{\circ} \mathrm{C}$.

\section{Cell Fusion Assays}

Infectious salmon anemia virus stably transfected HEK293, MDCK, and B16 cell lines were growth at 70-90\% confluence. Cell fusion was morphologically evaluated on a light microscope; 10 random field at $20 \times$ magnification were captured and analyzed using a CMOS camera (AmScope). To measure cell fusion in MDCK cells, we used CellTracker ${ }^{\mathrm{TM}}$ Green (CMFDA), which labels cytoplasm and nuclei, and CellTracker ${ }^{\mathrm{TM}}$ Red (CMTPX), which labels preferentially the cytoplasm. Briefly, MDCK cells were grown until $80 \%$ confluence, then the cells were incubated for $45 \mathrm{~min}$ at $37^{\circ} \mathrm{C}$ in non-supplemented DMEM containing $20 \mu \mathrm{M}$ CMFDA or $15 \mu \mathrm{M}$ CMTPX, washed, and then incubated for two additional hours. These cells were trypsinized and mixed in a 1:1 ratio (5,000 cells in total), and the suspension of mixed cells was seeded on $12 \mathrm{~mm}$ round coverslips and cultured for $24 \mathrm{~h}$. The cells were fixed in $1 \% \mathrm{w} / \mathrm{w}$ paraformaldehyde for $10 \mathrm{~min}$ at RT and mounted with 10\% DABCO (Sigma). The fluorescence images were acquired on a Zeiss LSM510 confocal microscope and processed using the Zeiss LSM 4 Image Browser software.

\section{Dendritic Cell Maturation}

Dendritic cell maturation was induced at day 7 post differentiation by adding $1.0 \mu \mathrm{g} / \mathrm{mL}$ LPS from $E$. coli 026:B6 (Sigma) or CBs in $0.5: 1,1: 1$, and $2: 1$ ratios (CBs/dendritic cells) for $24 \mathrm{~h}$. After the challenge, the cells were collected and labeled with the following antibodies: anti-mouse-CD11c-PE (clone N418), anti-mouseCD86-APC (clone GL1), anti-mouse-MHC class II- FITC (clone M5/114.15.2), anti-mouse/rat CD40-FITC (clone HM40-3), and anti-mouse-MHC class I-FITC (clone AF6-88.5.5.3) (eBioscience). The samples were analyzed by flow cytometry using the 
Accuri C6 Flow Cytometer (BD Bioscience), and the data were processed with the CFlow Plus software.

\section{Cross-Presentation in Dendritic Cells}

Dendritic cells were stimulated for $24 \mathrm{~h}$ with HEK293-OVA or HEK-OVA-ISAV CBs in a 1:1 and 2:1 ratio (CBs/dendritic cells). As a positive control, the cells were pulsed with $5 \mu \mathrm{M}$ OVA257264 derivative peptide (SIINFEKL, S7951, Sigma) incubated for $3 \mathrm{~h}$ at $37^{\circ} \mathrm{C}$ in a humidified atmosphere under $5 \% \mathrm{CO}_{2}$. After this time, cells were labeled with anti-mouse-CD11c and anti-mouse $\mathrm{OVA}_{257-264}$ (SIINFEKL/H-2Kb, clone eBio25-D1.16, eBioscience). The cells were analyzed by flow cytometry.

\section{Cytoplasmic Antigen Transfer Assay}

HEK293-ISAV stably transfected or wild-type cells were grown until $80 \%$ confluence on $100 \mathrm{~mm}$ dishes, incubated with $20 \mu \mathrm{M}$ CMFDA, and CBs were prepared as described above. Dendritic cells or Raw264.7 cells were incubated with $10 \mu \mathrm{M}$ CMTPX or $15 \mu \mathrm{M}$ CellTracker ${ }^{\mathrm{TM}}$ (CMRA), respectively, for $45 \mathrm{~min}$, and then they were challenged with fluorescent CBs for $24 \mathrm{~h}$. Cells were fixed in $1 \%$ PFA for $10 \mathrm{~min}$ and incubated with DAPI $(0.5 \mu \mathrm{g} / \mu \mathrm{L})$ for $1 \mathrm{~min}$ for confocal microscope analysis. For Raw264.7 and DCs, we quantified the CBs by measuring the mean fluorescence intensity in the cytoplasm using ImageJ (20).

\section{Antitumor Treatment on Melanoma Cancer Model}

To assess the effect of immunization with fusogenic CBs, we used a melanoma model (B16 cells). For preventive treatment, C57BL/6J mice were immunized with $100 \mu \mathrm{L}$ of CBs (from $1 \times 10^{5}$ cells) every 7 days for 3 weeks generated from wild-type B16 or fusion protein ISAV-transfected cells (B16-ISAV). CBs were injected subcutaneously in the abdominal region, far from the tumor growth area. One week after the final immunization, the mice were challenged with $2 \times 10^{5}$ viable B16 cells. In parallel, we evaluated the curative effect of fusogenic CBs on melanoma. Briefly, the mice were injected subcutaneously in the lumbar region with $2 \times 10^{5}$ viable B16 cells (C57BL/6J), and 7, 14, and 21 days after the challenge they were inoculated with $100 \mu \mathrm{L}$ of CBs (from $1 \times 10^{5}$ cells) generated from wild-type or ISAV-B16 transfected cells. For both treatment protocols, tumor appearance and size were monitored daily, when the tumor reached a volume of $250 \mathrm{~mm}^{3}$ (calculated by a semi-sphere formula $V\left[\mathrm{~mm}^{3}\right]=2 / 3 \pi^{\star} r^{3}$ ) mice were sacrificed and the spleen and tumor were removed.

To evaluate different lymphocyte populations, the spleen of treated mice was disaggregated on a $100 \mu \mathrm{m}$ metal mesh and erythrocytes were eliminated by differential lysis, using ACK buffer (10 mM $\mathrm{KHCO}_{3}, 155 \mathrm{mM} \mathrm{NH}_{4} \mathrm{Cl}, 1 \mathrm{mM}$ EDTA, pH: 7.3). For the tumor-infiltrating lymphocyte (TIL), the tumors were cut into small pieces, disaggregated, and suspended using a Tumor Dissociation Kit (MiltenyiBiotec Inc.). Splenocytes and tumor cells were suspended at a density of $1 \times 10^{6} \mathrm{cell} / \mathrm{mL}$ in cold blocking buffer (IF; $2 \% \mathrm{FBS}$ in PBS) for $30 \mathrm{~min}$ at $4^{\circ} \mathrm{C}$. The following antibodies were used to identify the lymphocyte populations by flow cytometry: anti-mouse CD4 FITC (cat. 11-0042) and antiCD8 Ly-2 PE (cat. 553033) (BD Biosciences).

\section{Statistical Analyses}

Tumor appearance was evaluated by the Kaplan-Meier method. Tumor growth was analyzed by multiple $t$-test followed by HolmSidak's method. Cell fusion induced by ISAV fusion protein and antigen transfer was analyzed using the Mann-Whitney test. The percentage of CD8+, CD4+, Tregs, Th1, and Th17 lymphocytes was analyzed using Kruskal-Wallis ANOVA. For all experiments, statistical differences between groups and treatments were considered significant when $p<0.05$. All statistical analyses were performed using GraphPad Prim version 5.

\section{RESULTS}

\section{ISAV Fusion Protein Keeps Its Fusogenic Capability in Mammalian Cells}

To determine whether ISAV fusion protein retains its fusogenic activity when expressed in mammalian cells, HEK293 and MDCK cells were stably transfected with the open reading frame of ISAV segment 5, which corresponds to the fusogenic protein (fusion protein ISAV). We found that after $48-72 \mathrm{~h}$ post-passage, non-transfected HEK293 and transfected HEK293-ISAV showed morphological changes associated with cell fusion (Figure 1A); however, HEK293-ISAV presents a higher number of fused cells (HEK293 $=0.38 \pm 0.23$ vs. HEK293-ISAV $=1.08 \pm 0.26$; Figure 1B). In addition, we evaluated cell fusion in MDCK cells because this cell line has a polygonal morphology with an ordered epithelial monolayer, and the limits between cells are easily distinguishable. We found that ISAV fusion protein induces fusion in MDCK, showing zones with an increased cell size, loss cell limits, and loss of the polygonal shape (Figure 1C). The quantification showed that MDCK expressing ISAV fusion protein showed a higher number of fused cells $(\mathrm{MDCK}=0.08 \pm 0.083$ vs. MDCK$\mathrm{ISAV}=1.13 \pm 0.38$; Figure 1D).

To test the action of the ISAV fusion protein on cell fusion, MDCK cells were labeled with CMTPX or CMFDA. Nontransfected MDCK cells showed only a single fluorophore labeling, with no overlapping (Figure 1E, top panel). However, MDCK-ISAV showed labeling of both fluorophores, a cytoplasmic red labeling and a nuclear and cytoplasmic green labeling (Figure 1E, bottom panel). These results indicate that ISAV fusion protein allows the transfer of the fluorophores between cells, indicating a process of cellular fusion.

\section{ISAV Fusion Protein Expression on CBs Allow Antigen Transfer by Increasing Its Internalization in APCs}

Once we confirmed that the ISAV fusion protein keeps its fusogenic capability in mammalian cells, we evaluated the difference in the internalization levels of CBs generated from HEK293 and HEK293-ISAV using Raw264.7 and BM-dendritic cells. Both cell types challenged with HEK293 CBs showed low levels of internalization; in contrast, cells challenged with CBs from HEK293-ISAV showed larger numbers of cells associated with CBs (Figures 2A,C). These results show that the ISAV fusion protein expression on CBs derived from HEK293 cells produce 
A
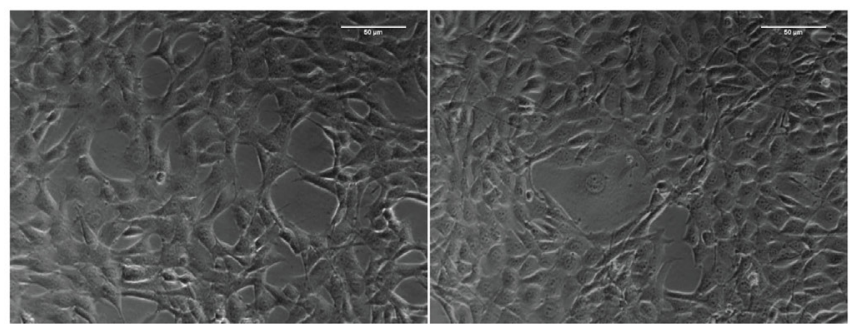

C

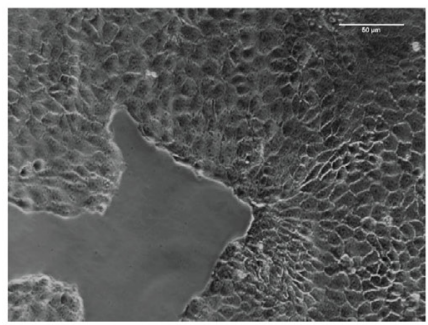

B

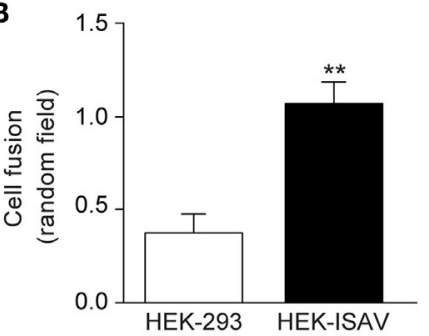

D

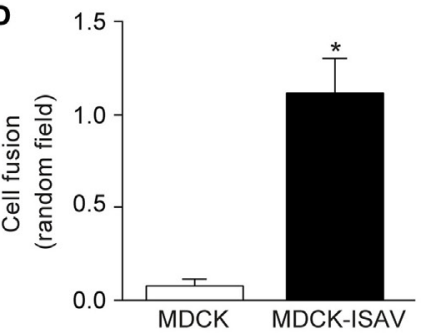

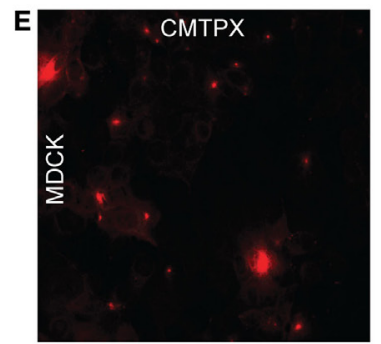
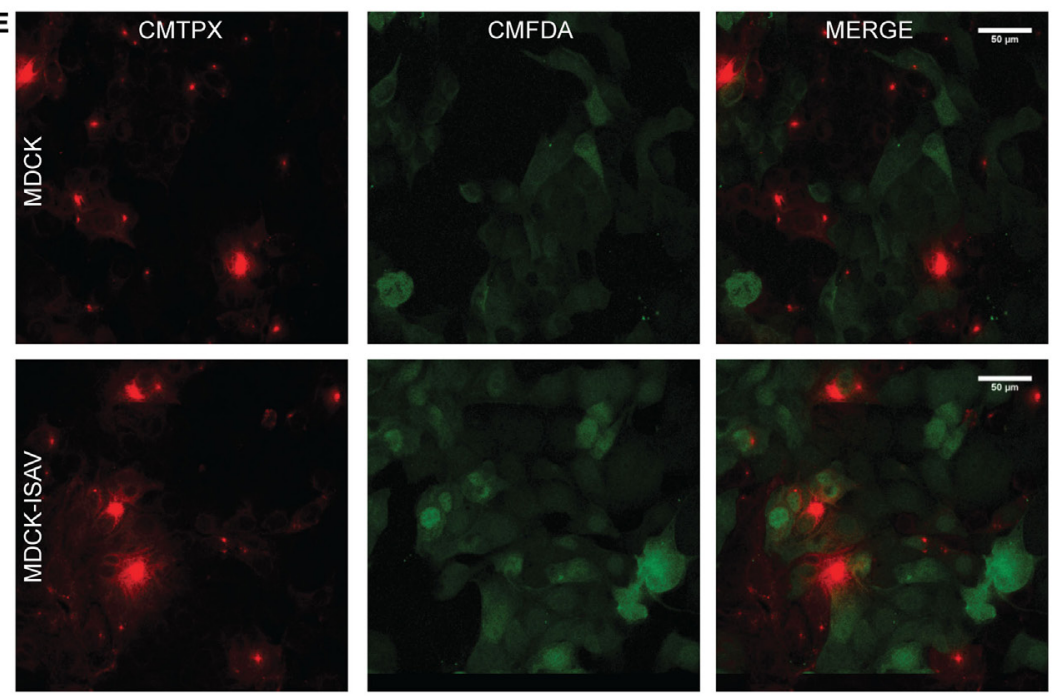

FIGURE 1 | Fusion protein infectious salmon anemia virus (ISAV) induces fusion in HEK293 and MDCK cells. (A) Light microscope images of cell fusion in non-transfected (left) or ISAV-transfected HEK293 cells (right), (B) quantification of cell fusion of 10 random fields in non-transfected or ISAV-transfected HEK293 cells $(n=5)$. (C) Light microscope images of cell fusion in non-transfected (left) or ISAV-transfected MDCK cells (right). (D) Quantification of cell fusion of 10 random fields in non-transfected or ISAV-transfected MDCK cells $(n=5)$. (E) MDCK cells were labeled with CMFDA or CMTPX separately, and then they were mixed and grown for $24 \mathrm{~h}$. The upper panel shows non-transfected MDCK and the lower panel shows ISAV-transfected MDCK. Statistical analyses were performed using the Mann-Whitney test $\left({ }^{*} p<0.05,{ }^{* *} p<0.01\right)$.

an efficient antigen internalization and transfer in both Raw264.7 (Figure 2B) and dendritic cells (Figure 2D) as compared with ISAV non-expressing cells.

\section{ISAV Fusion Protein Does Not Interfere with Dendritic Cell Maturation and Cross-Presentation Induced by CBs}

To characterize the effect of CBs on dendritic cell maturation, dendritic cells were challenged with different ratios of $\mathrm{CBs}$ (0.5:1, 1:1, and 2:1 CBs/DCs). The evaluation of surface markers of maturation showed a low expression of CD86 and CD40 co-stimulatory molecules in non-stimulated dendritic cells (Figures 3A,B, respectively). On the other hand, dendritic cells stimulated with CBs from HEK293 and/or from HEK293-ISAV showed an increase in the expression of CD86 (Figure 3A) and CD40 (Figure 3B), but maturation levels were lower than in dendritic cells stimulated with LPS (positive control). Furthermore, we observed an increase in MHC-I (Figure 3C) and MHC-II (Figure 3D) expression in dendritic cells treated with CBs from HEK293 and HEK293-ISAV, and in the case of MHC-II reaching higher levels than LPS. We did not find differences in dendritic cells maturation with CBs from HEK293 and HEK293-ISAV. 

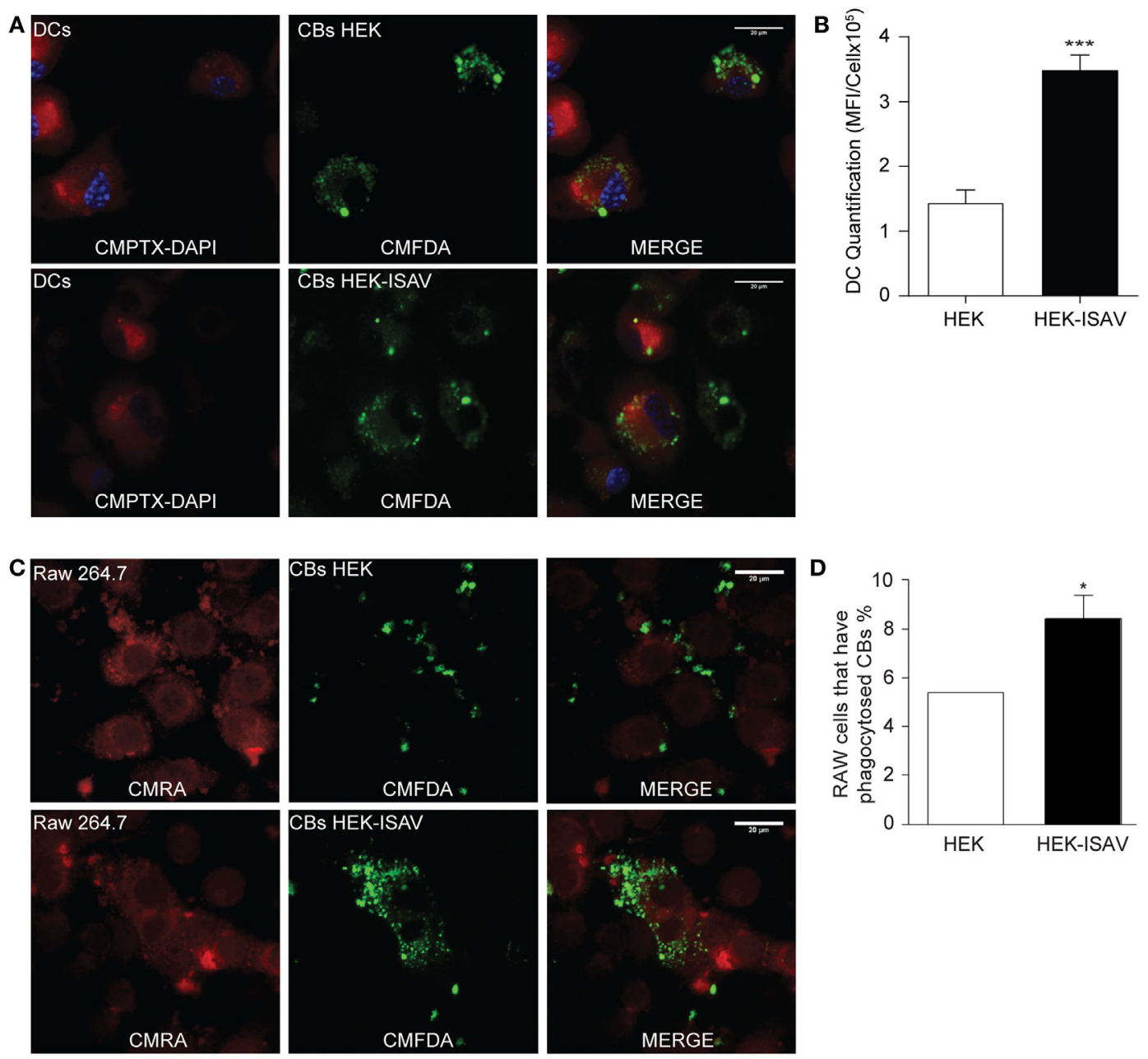

HEK

HEK-ISAV

FIGURE 2 | Fusion protein infectious salmon anemia virus (ISAV) facilitates antigen transfer to antigen presenting cells. (A) Phagocytosis of fluorescent cell bodies (CBs) by dendritic cells; the top panel shows dendritic cells in the presence of non-transfected CBs; the bottom panel shows dendritic cells in the presence of CBs transfected with ISAV. (B) Quantification of mean fluorescence intensity of CBs in the cytoplasm of DCs (C) Phagocytosis of fluorescent CBs by Raw.267; the top panel shows Raw.267 in the presence of non-transfected CBs, the bottom panel shows Raw.267 in the presence of CBs transfected with ISAV. (D) Quantification of RAW.264.7 cells percent that have phagocytosed fluorescent CBs $(n=7)$. Statistical analyses were performed using the Mann-Whitney test $\left({ }^{*} p<0.05\right.$, $\left.{ }^{\star \star *} p<0.001\right)$.

To determine the effect of fusogenic CBs on cross-presentation, we generated CBs from HEK293 that expressed OVA antigen and the fusion protein ISAV (confirmed by RT-PCR). Dendritic cells challenged with HEK293-ISAV-OVA CBs showed a higher level of MHC-I/SIINFEKL than dendritic cells incubated with CBs from HEK293-OVA. However, none of them reached the levels of expression of dendritic cells loaded with OVA257-26 peptide (SIINKFEL) (Figure 3E). Altogether, these results show that the expression of fusion protein ISAV in cellular bodies does not interfere with dendritic cells maturation neither cross-presentation.

\section{Treatment with CBs Delays B16 Melanoma Tumor Growth}

While cellular bodies induce maturation of dendritic cells, we sought to determine whether CBs induce an antitumor immune response when they are used as a treatment. First, we evaluated the cell fusion capability of ISAV fusion protein using a syngeneic melanoma model (B16). In this experiment, we found that ISAV protein induces cell fusion in B16 cells (Figure 4A). Next, we evaluated the dendritic cell maturation in response to different ratios of cellular bodies generated in the B16 and B16-ISAV cell lines, this treatment increases the expression of CD86 (Figure 4B), CD40 (Figure 4C), MHC-I (Figure 4D), and MHC-II (Figure 4E) in dendritic cells in a 1:1 and 2:1 ratio (CBs/DCs) showing that both $\mathrm{CBs}$ induced dendritic cells maturation.

To determine the effect of $\mathrm{CBs}$ on the growth of established tumors, we generated tumors from B16 cells on C57BL/6 mice (Figure 5A). In the B16 melanoma tumor model, the treatment with $\mathrm{CBs}$ and ISAV CBs do not change the tumor growth (Figure 5B), with a significant decrease in CD8 TILs (Figure 5C) on B16-ISAV treated mice. To address whether both CBs treatment were linked to a systemic response, we assessed the CD4 and CD8 cell populations in the spleen. We found no changes in CD4 and CD8 populations (Figure 5D), in response to treatment compared to untreated animals. 


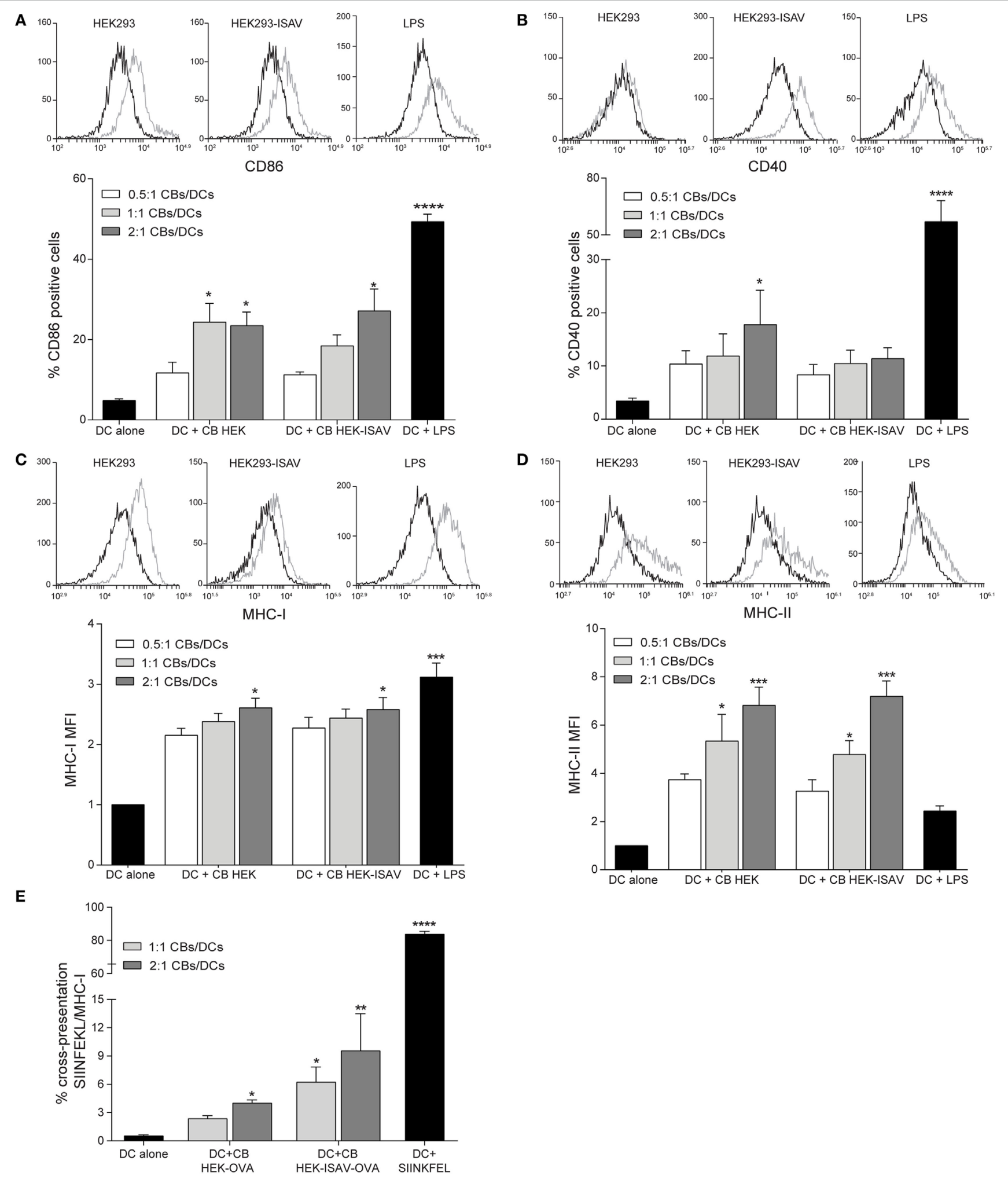

FIGURE 3 | HEK293 cell bodies (CBs) induce dendritic cell maturation. Dendritic cells were challenged with different ratios of CBs derived from non-transfected and infectious salmon anemia virus (ISAV)-transfected HEK-293 cells for $24 \mathrm{~h}$. Maturation markers were measured by flow cytometry, for each marker, a representative histogram of the ratio 1:1 [CBs/DCs] is shown (control, black line; stimulated, gray line). (A) \% CD86 and (B) \% CD40 positive cells. (C) MHC-I and (D) MHC-II mean fluorescence intensity (MFI) $(n=5)$. (E) Cross-presentation was measured by the detection of SIINFEKL-MHC-I $(n=6)$. Statistical analyses were performed using Kruskal-Wallis ANOVA $\left({ }^{\star} p<0.05,{ }^{* \star} p<0.005,{ }^{* \star *} p<0.001\right)$. 
A

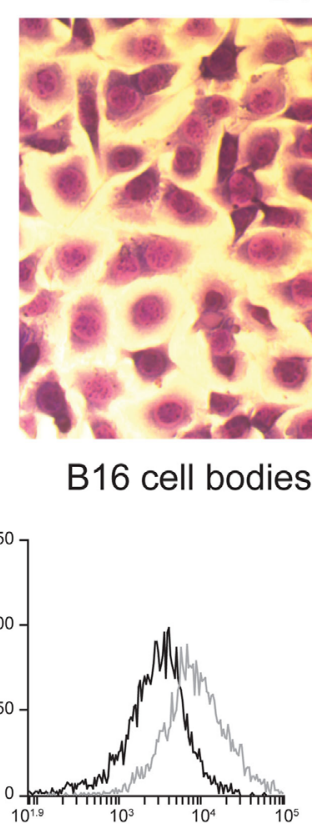

C

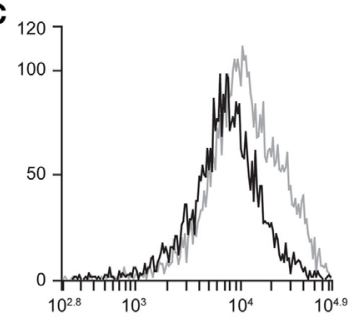

D

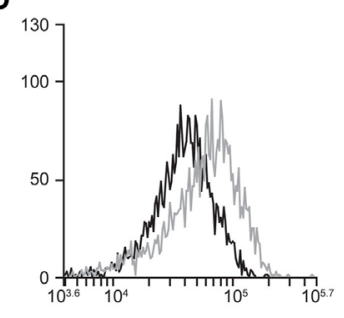

E

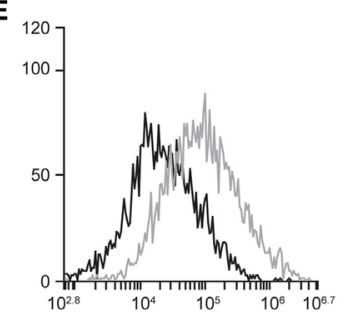

B16

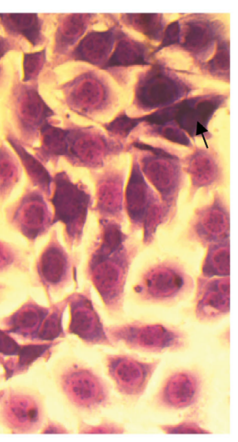

B16-ISAV cell bodies
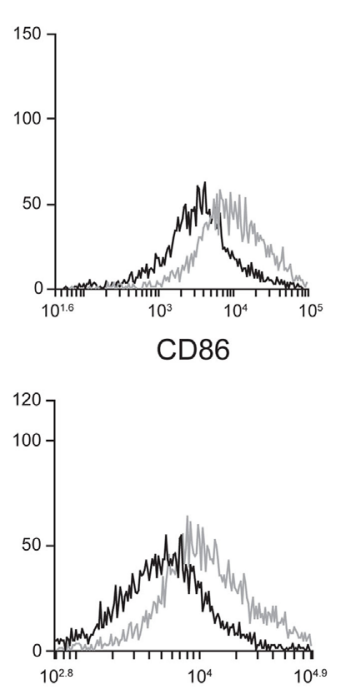

CD40
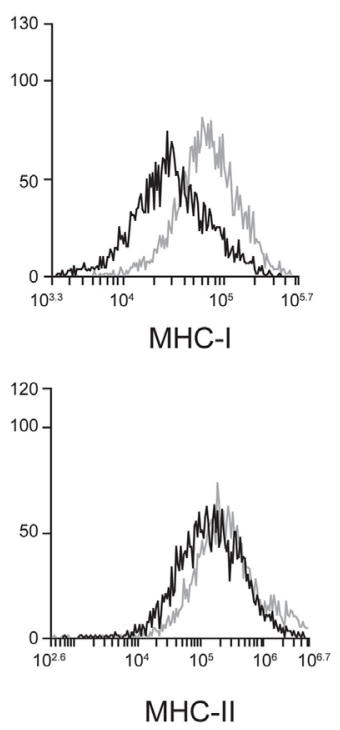
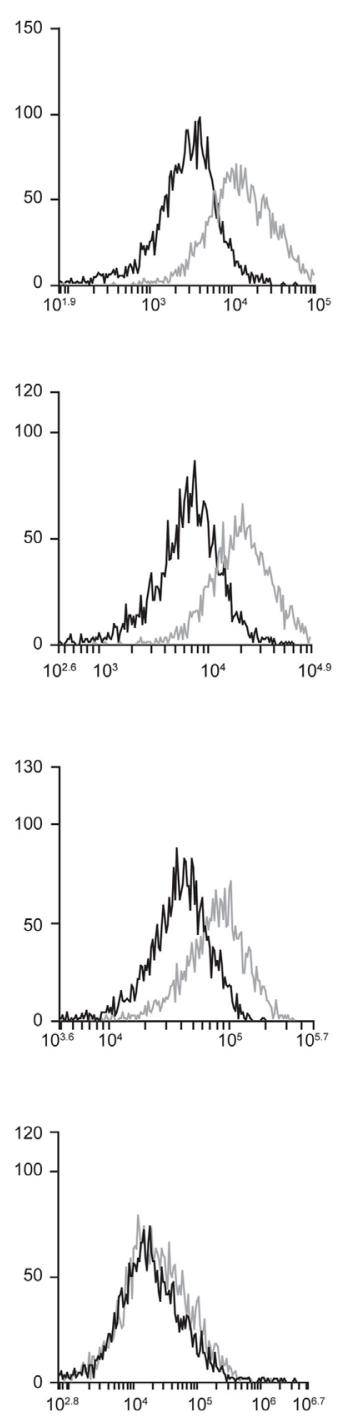

FIGURE 4 | Fusion protein infectious salmon anemia virus (ISAV) induces fusion in B16, and cell bodies (CBs) derived from B16 and B16-ISAV induce dendritic cell maturation. (A) Cell fusion was evaluated by transmitted light microscopy and hemacolor stain in non-transfected and ISAV-transfected cells B16 (arrows indicate fused cells). The expression of maturation markers determined by the percentage of (B) CD86 and (C) CD40 positive cells, and by the mean fluorescence intensity for (D) MHC-I and (E) MHC-II, induced by CBs was measured in dendritic cells by flow cytometry. Representative histograms of the ratio 1:1 (CBs/DCs) of dendritic cells stimulated with B16 and B16-ISAV bodies (control, black line; stimulated, gray line). 


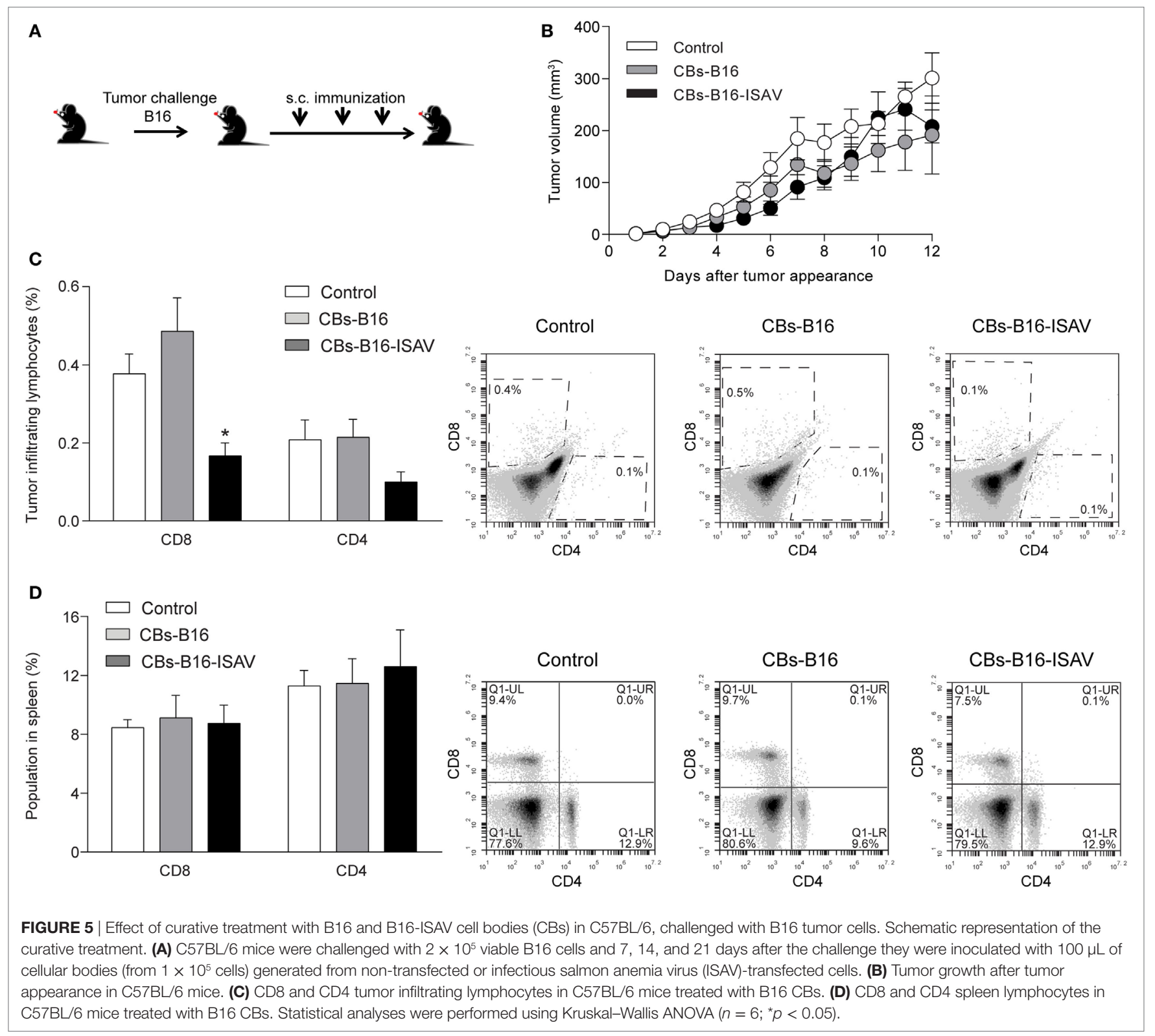

To evaluate whether tumor growth masked the effect of the curative treatment on established tumors, we determine the effect of the previous immunization on tumor development using both types of CBs in C57BL/6 mice (Figure 6A). We found that mice immunized with CBs from B16 and B16-ISAV did not show a delay in tumor appearance (Figure 6B) but instead it showed a delay in tumor growth (Figure 6C). The immunization with B16ISAV CBs was not correlated with changes in the CD8 and CD4 populations in the tumor (Figure 6D), but it was correlated with an increase of the CD8 and CD4 populations in spleen (Figure 6E).

\section{DISCUSSION}

Immunotherapy is a promising tool for treatments against cancer, numerous studies demonstrated that this type of therapy can prevent or stop tumor growth. Although this therapy has brought satisfactory results, its cost has undermined their clinical use. Recent evidence suggests that chemotherapeutical agents can induce ICD and triggers an immune response, thus participating in the eradication of the tumor. In this work, we tested the effect of antigen-loaded CBs expressing ISAV fusion protein generated in vitro (which mimics ICD) as an immunotherapy against melanoma. Using a prophylactic treatment paradigm, we observed a delay in tumor growth. Furthermore, CBs stimulate dendritic cells maturation and induce antigen cross-presentation in vitro.

The effective activation of an antitumor immune response requires the delivery of antigens to dendritic cells inducing cross-priming and an efficient activation of reactive $\mathrm{T}$ cells. In this context, the use of whole tumor cells as an antigen source offers an advantage by its high diversity of undefined antigens, 


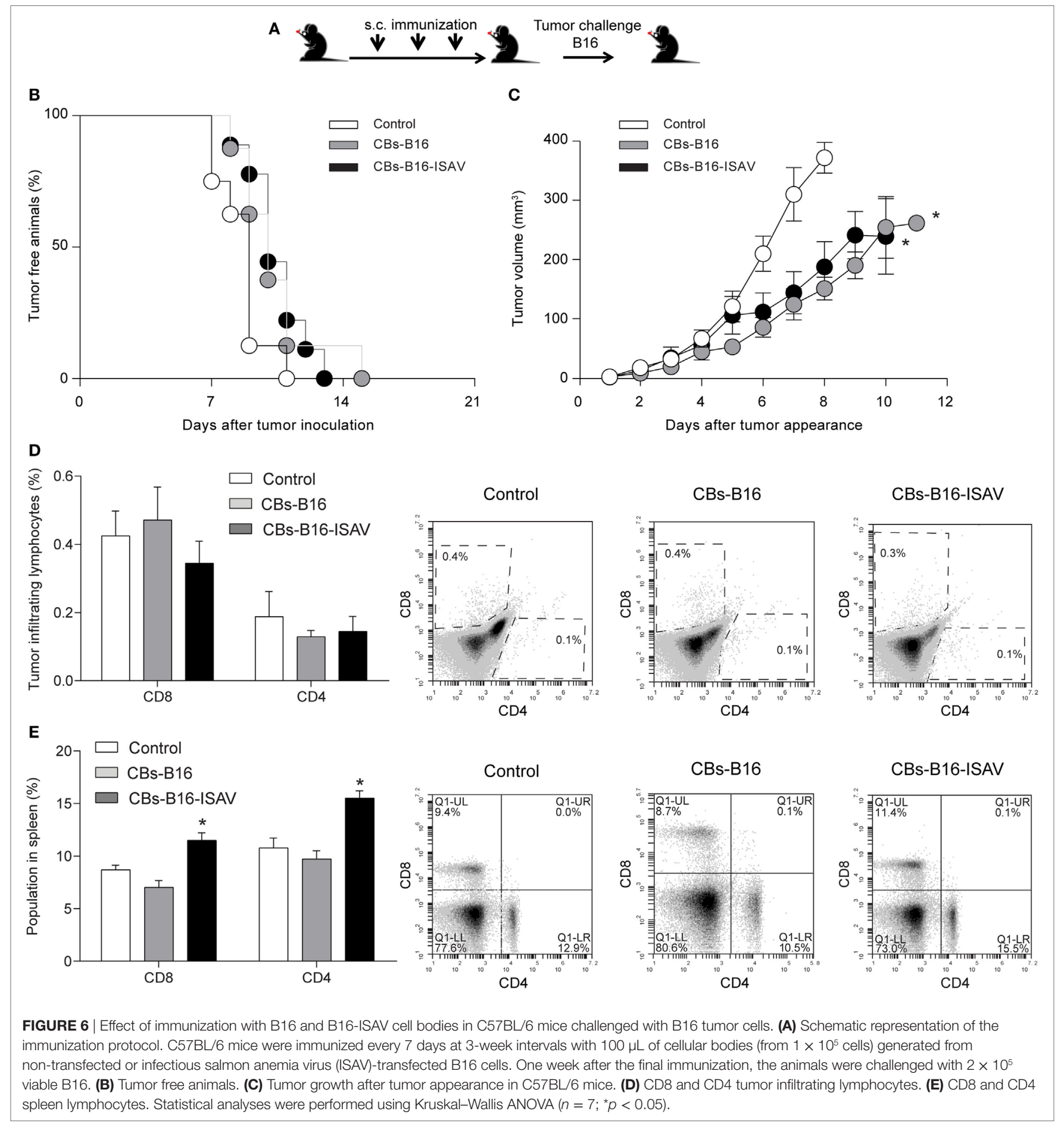

increasing the chances to mount a robust immune response (21). For instance, dendritic cell vaccines pulsed with apoptotic CBs (derived from whole tumor cells) induce a more efficient antitumor immune response (22) than dendritic cell vaccines pulsed with tumor lysates (23). In this regard, our results show that activated dendritic cells can phagocytize apoptotic CBs, suggesting that apoptotic $\mathrm{CBs}$, as a source of antigens, may improve antigen transference into dendritic cells.
In salmon cells, ISAV-F protein stimulates cell fusion in the absence of accessory glycoproteins (24). Using ISAV-F protein, we observed cell fusion in mammalian cells lines such as HEK293, 4T1 (data not showed), B16 and MDCK. Moreover, cells bodies expressing ISAV-F protein favors antigen delivery into dendritic cells in a mechanism related to the fusion between dendritic cells and CBs membranes [similar to the study by Koido et al. (25)], this is not correlated with differences in dendritic cell maturation 
and antigen cross-presentation. In contrast, dead cells expressing fusion proteins in its surface display an increased cross-presentation (26). Our result could be explained because the mammals are not the natural hosts of ISA virus and the ISAV-F protein activity could be different at the $\mathrm{pH}$ (7.4 vs. 5.4) and temperature (37 vs. $14^{\circ} \mathrm{C}$ ) tested (24).

Our results demonstrated that $\mathrm{CBs}$ induce dendritic cell maturation per se, this effect could be related to the autophagy induced by nutrient deprivation (27), which induce the expression of High-Mobility Group Box 1 nuclear protein, calreticulin translocation, and the release of degradation products such as ATP (28). All of these components are well-known damage-associated molecular patterns (DAMPs), which activate dendritic cell (29). Our studies indicate that our method to produce CBs induces ICD, and these CBs express DAMPs (Lopez et al., manuscript in preparation).

We observed that prophylactic treatment using CBs from $\mathrm{B} 16$ and B16-ISAV generates a delay in tumor growth in B16 melanoma model, but only the treatment with B16-ISAV CBs showed an increase in systemic CD8+ and CD4+ lymphocytes which are associated with an antitumor response. Nonetheless, in both treatments (prophylactic and curative), we did not observe variations in CD8 and CD4 TILs population and we only found a reduction in the CD8 TIL in the curative treatment. The interpretation of these results requires the precise analysis of CD45+ population to determine the extent of CD8+ and CD4+ TILs variations. Additionally, the lack of effect in lymphocytes population may be explained by the timing of the population analysis of the spleen and tumor, these were performed in the late phase of the tumor growth, when the tumor already surpassed the immune system by inducing a tolerogenic response. In this regard, it will be necessary to measure the population at initial stages of growth to obtain the precise kinetic of the populations during the immune response against the tumor.

\section{REFERENCES}

1. Yewdell JW. Designing CD8+ T cell vaccines: it's not rocket science (yet). Curr Opin Immunol (2010) 22:402-10. doi:10.1016/j.coi.2010.04.002

2. Geissmann F, Manz MG, Jung S, Sieweke MH, Merad M, Ley K. Development of monocytes, macrophages and dendritic cells. Science (2010) 327:656-61. doi:10.1126/science.1178331

3. Shimizu K, Kuriyama H, Kjaergaard J, Lee W, Tanaka H, Shu S. Comparative analysis of antigen loading strategies of dendritic cells for tumor immunotherapy. J Immunother (2004) 27:265-72. doi:10.1097/00002371-200407000-00002

4. Andre F, Escudier B, Angevin E, Tursz T, Zitvogel L. Exosomes for cancer immunotherapy. Ann Oncol (2004) 15(Suppl 4):iv141-4. doi:10.1093/annonc/ mdh918

5. Chaput N, Taïeb J, Schartz NEC, André F, Angevin E, Zitvogel L. Exosomebased immunotherapy. Cancer Immunol Immunother (2004) 53:234-9. doi:10.1007/s00262-003-0472-x

6. Krysko DV, Garg AD, Kaczmarek A, Krysko O, Agostinis P, Vandenabeele P. Immunogenic cell death and DAMPs in cancer therapy. Nat Rev Cancer (2012) 12:860-75. doi: $10.1038 / \mathrm{nrc} 3380$

7. Vacchelli E, Aranda F, Eggermont A, Galon J, Sautès-Fridman C, Cremer I, et al. Trial watch: chemotherapy with immunogenic cell death inducers. Oncoimmunology (2014) 3:e27878. doi:10.4161/onci.27878

8. Casares N, Pequignot MO, Tesniere A, Ghiringhelli F, Roux S, Chaput N, et al. Caspase-dependent immunogenicity of doxorubicin-induced tumor cell death. J Exp Med (2005) 202:1691-701. doi:10.1084/jem.20050915
Altogether, our findings suggest the use of CBs expressing ISAV fusion protein induce dendritic cells maturation in vitro, antigen transfer by macrophages and dendritic cells, and an increased cross-presentation by dendritic cells. Despite the delay in tumor growth, this effect is not related to a resolving immune response. Overall, our results suggest that CBs can be used as a complement with other strategies to amplify the antitumor immune response.

\section{ETHICS STATEMENT}

All procedures were conducted in accord to guidelines on the recognition of pain, distress, and discomfort in experimental animals described by Morton and Griffiths, except for temperature evaluation. Protocols were reviewed and approved by the Ethics Committee of the Universidad de Santiago de Chile.

\section{AUTHOR CONTRIBUTIONS}

JM, CC, CB, and DR: phagocytosis assay, antigen transference, and fusion experiments. SC, CR-P, XL, and ET: molecular biology. CB, CC, and CB-A: dendritic cell experiments. MI and MM: experimental molecular biology design. MC-SM, LR, and DS experimental immunological design. AE, RF, EL-S, DR, CR-P, and CA-C: experimental design and wrote the paper.

\section{ACKNOWLEDGMENTS}

This study was funded by FONDECYT 1110734 and 1161015 (MI), 11140915 (LR), 11140731 (EL-S), PAI 79140059 (EL-S), DICYT and DGT s/n (CA-C), and CONICYT fellowship (CR-P, CB-A).

9. Gannagé M, Münz C. Autophagy in MHC class II presentation of endogenous antigens. Curr Top Microbiol Immunol (2009) 335:123-40. doi:10.1007/978-3-642-00302-8_6

10. MacLean G, Li H, Metzger D, Chambon P, Petkovich M. Apoptotic extinction of germ cells in testes of Cyp26b1 knockout mice. Endocrinology (2007) 148:4560-7. doi:10.1210/en.2007-0492

11. Li Y, Wang L-X, Yang G, Hao F, Urba WJ, Hu H-M. Efficient cross-presentation depends on autophagy in tumor cells. Cancer Res (2008) 68:6889-95. doi:10.1158/0008-5472.CAN-08-0161

12. Li Q-X, Liu G, Wong-Staal F. Oncolytic virotherapy as a personalized cancer vaccine. Int J Cancer (2008) 123:493-9. doi:10.1002/ijc.23692

13. Inoue $\mathrm{H}$, Tani $\mathrm{K}$. Multimodal immunogenic cancer cell death as a consequence of anticancer cytotoxic treatments. Cell Death Differ (2014) 21:39-49. doi:10.1038/cdd.2013.84

14. Bartlett DL, Liu Z, Sathaiah M, Ravindranathan R, Guo Z, He Y, et al. Oncolytic viruses as therapeutic cancer vaccines. Mol Cancer (2013) 12:103. doi:10.1186/1476-4598-12-103

15. Hoffmann D, Bayer W, Wildner O. Therapeutic immune response induced by intratumoral expression of the fusogenic membrane protein of vesicular stomatitis virus and cytokines encoded by adenoviral vectors. Int J Mol Med (2007) 20:673-81.

16. Linardakis E, Bateman A, Phan V, Ahmed A, Gough M, Olivier K, et al. Enhancing the efficacy of a weak allogeneic melanoma vaccine by viral fusogenic membrane glycoprotein-mediated tumor cell-tumor cell fusion. Cancer Res (2002) 62:5495-504. 
17. Stegmann T. Membrane fusion mechanisms: the influenza hemagglutinin paradigm and its implications for intracellular fusion. Traffic (2000) 1:598-604. doi:10.1034/j.1600-0854.2000.010803.x

18. Morton D, Griffiths P. Guidelines on the recognition of pain, distress and discomfort in experimental animals and an hypothesis for assessment. Vet Rec (1985) 116:431-6. doi:10.1136/vr.116.16.431

19. Inaba $K$, Inaba $M$, Romani $N$, Aya $H$, Deguchi $M$, Ikehara S, et al. Generation of large numbers of dendritic cells from mouse bone marrow cultures supplemented with granulocyte/macrophage colony-stimulating factor. J Exp Med (1992) 176:1693-702. doi:10.1084/jem.176.6.1693

20. McCloy RA, Rogers S, Caldon CE, Lorca T, Castro A, Burgess A. Partial inhibition of Cdk1 in G2 phase overrides the SAC and decouples mitotic events. Cell Cycle (2014) 13(9):1400-12. doi:10.4161/cc.28401

21. Errington F, Jones J, Merrick A, Bateman A, Harrington K, Gough M, et al. Fusogenic membrane glycoprotein-mediated tumour cell fusion activates human dendritic cells for enhanced IL-12 production and T-cell priming. Gene Ther (2006) 13:138-49. doi:10.1038/sj.gt.3302609

22. Goldszmid RS, Idoyaga J, Bravo AI, Steinman R, Mordoh J, Wainstok R. Dendritic cells charged with apoptotic tumor cells induce long-lived protective CD4+ and CD8+ T cell immunity against B16 melanoma. J Immunol (2003) 171:5940-7. doi:10.4049/jimmunol.171.11.5940

23. Scheffer SR, Nave H, Korangy F, Schlote K, Pabst R, Jaffee EM, et al. Apoptotic, but not necrotic, tumor cell vaccines induce a potent immune response in vivo. Int J Cancer (2003) 103:205-11. doi:10.1002/ijc.10777

24. Aspehaug V, Mikalsen AB, Snow M, Biering E. Characterization of the infectious salmon anemia virus fusion protein. J Virol (2005) 79:12544-53. doi:10.1128/JVI.79.19.12544-12553.2005

25. Koido S, Homma S, Okamoto M, Namiki Y, Takakura K, Uchiyama K, et al. Strategies to improve the immunogenicity of anticancer vaccines based on dendritic cell/malignant cell fusions. Oncoimmunology (2013) 2:e25994. doi:10.4161/onci.25994

26. Bateman AR, Harrington KJ, Kottke T, Ahmed A, Melcher AA, Gough MJ, et al. Viral fusogenic membrane glycoproteins kill solid tumor cells by nonapoptotic mechanisms that promote cross presentation of tumor antigens by dendritic cells. Cancer Res (2002) 62:6566-78.

27. Hou W, Zhang Q, Yan Z, Chen R, Zeh HJ III, Kang R, et al. Strange attractors: DAMPs and autophagy link tumor cell death and immunity. Cell Death Dis (2013) 4:e966. doi:10.1038/cddis.2013.493

28. Yi Y, Zhou Z, Shu S, Fang Y, Twitty C, Hilton TL, et al. Autophagy-assisted antigen cross-presentation: autophagosome as the argo of shared tumor-specific antigens and DAMPs. Oncoimmunology (2012) 1:976-8. doi:10.4161/onci.20059

29. Halstead JM, Lionnet T, Wilbertz JH, Wippich F, Ephrussi A, Singer RH, et al. Translation. An RNA biosensor for imaging the first round of translation from single cells to living animals. Science (2015) 347:1367-671. doi:10.1126/ science.aaa3380

Conflict of Interest Statement: The authors declare that the research was conducted in the absence of any commercial or financial relationships that could be construed as a potential conflict of interest.

Copyright (c) 2017 Morales, Barrera-Avalos, Castro, Castillo, Barrientos, RoblesPlanells, López, Torres, Montoya, Cortez-San Martín, Riquelme, Escobar, Fernández, Imarai, Sauma, Rojo, Leiva-Salcedo and Acuña-Castillo. This is an open-access article distributed under the terms of the Creative Commons Attribution License (CC BY). The use, distribution or reproduction in other forums is permitted, provided the original author(s) or licensor are credited and that the original publication in this journal is cited, in accordance with accepted academic practice. No use, distribution or reproduction is permitted which does not comply with these terms. 